\title{
Study of IL-33 and IL-1R4 in Iraqi Rheumatoid Arthritis Female Patient's with and without Dyslipidemia Prone to Atherosclerosis.
}

\author{
Lara Ali Nazar \\ laraali398@gmail.com \\ EimanAA.Abass \\ drbiochem2007@gmail.com \\ Department of Chemistry,College of Education for Pure Science Ibn Al-Haitham, University of \\ Baghdad,Baghdad,Iraq.
}

Article history: Received 31 August 2018, Accepted 17 October 2018, Publish January 2019

\begin{abstract}
This study is planned to find relationship between interleukin-33 (IL-33) with its receptor interleukin-1 receptor 4 (IL-1R4), and assurance IL-33/IL-1R4 proportion as biomarker to atherosclerosisin rheumatoid arthritis (RA) Iraqi female's patients with and without dyslipidemia. This study was attempted at Baghdad Teaching Hospital included 60 female's patients with RA that were isolated into: 30 patients with dyslipidemia(G2), 30 patients without dyslipidemia(G3) and 30 individuals as control group (G1). Patients were experiencing treatment by methortexiene medication, analyzed by rheumatoid factor (RF) and erythrocyte sedimentation rate (ESR) tests. All patients and control groups age ranged from (30-55) years. The results show an increase in ESR, RF, IL-33, and IL-1R4 levels. In addition to decrease in IL-33/IL-1R4 ratio in the two patient's groups when contrasted and control group. The momentum examine inferred that the level of ESR, and IL-33 in RA Iraqi females patients with dyslipidemia were higher than that in RA Iraqi females patients without dyslipidemia, while the level of IL-33/IL-1R4 ratio in RA Iraqi females patients with dyslipidemia was lower than that in RA Iraqi females patients without dyslipidemia patients; in this manner the IL-33/IL-1R4 ratio may be used as a biomarker in diagnostic early porn to atherosclerosis in RA females patients with dyslipidemia.
\end{abstract}

keywords: Rheumatoid arthritis, IL-33, IL-1R4, atherosclerosis disease, ESR and RF.

\section{Introduction}

Rheumatoid arthritis is a constant, incendiary, portrayed by joint torment, swelling, deformation, and eventually, handicap. Lymphocytes intercedes the safe issue in RA. Various cell writes take an interest in the pathogenesis. The exact pathogenic instrument isn't completely comprehended [1]. RA is a standout amongst the most predominant fundamental incendiary illnesses which include joints and additional articular tissues, subsequently causing organ harm. In light of the endless irritation and invulnerable dysregulation, the nearness of RA has been related with cardiovascular (CV) disease and an expanded CV related mortality [2]. The correct reason for RA has not been recognized but rather a few investigations called attention to that genius fiery cytokines, including tumor necrosis factor (TNF)- $\alpha$, interleukin IL-1 family, IL-6, IL-17 and the arbiters delivered through downstream pathways in the ligament joints, constitute the milieu driving cartilage and bone destruction [3]. 
The IL-33 initiates its signaling pathway by binding to the suppression of tumorigenicity2 (ST2) receptor, or also known as IL-1R4. IL-33 is fundamentally associated with the T-helper cell type 2 (Th2) safe reaction acceptance which gives defensive safe reaction towards parasitic disease, specifically helminths [4]. IL-33 receptors are composed of ST2 (also known as IL-1R4), a ligand binding chain, and IL-1 receptor accessory protein (IL-1RAcP, also known as IL-1R3), a signal transducing chain. IL-1R3 is a common receptor for IL-1a, and IL-1b, IL-33, and three IL-36 isoforms [5].

The IL-1R4 is an individual from the interleukin 1 receptor family. ST2 remains for "suppression of tumorigenicity 2". It was found in 1989, yet just in 2002 Weinberg et al. revealed that it could be communicated via heart cells in light of myocardial pressure, drawing the consideration of scientists to a part in the cardiovascular framework. ST2 has two fundamental isoforms: Trans membrane or cell (ST2L) and dissolvable or circling (sST2) forms [6]. The association of IL-33 and ST2L has been turned out to be cardioprotective in exploratory models, lessening myocardial fibrosis, cardiomyocyte hypertrophy, apoptosis, and enhancing myocardial capacity. This cardioprotective activity happens only through the ST2L receptor and not through the solvent receptor. The IL-33/ST2 framework is upregulated in cardiomyocytes and fibroblasts in light of cardiovascular damage. The collaboration of this dissolvable receptor with IL-33 obstructs the IL-33/ST2L framework Thus, disposes of the cardio defensive impacts depicted previously. The ST2 framework acts not just as a middle person of IL-33 work in its transmembrane full-length ST2L isoform yet additionally as an inhibitor of IL-33 through its dissolvable soluble suppression of tumorigenicity 2(sST2) isoform [7].

The IL-33/ST2 has emerged as an intercellular signaling system that participates in processes as varied as the antigen/allergen response, autoimmunity, organ fibrosis and cardiac injury. Exploration of the effects of this pathway is underway. An effort to clarify the role of IL-33/ST2 in the crosstalk between end-organ effector cells and their environment, specifically the surrounding matrix and inflammatory cells, may lend itself to the discovery of novel therapeutic targets for the treatment of diseases such as asthma, rheumatoid arthritis, atherosclerosis and heart failure [8].

The ESR is a nonspecific measure of inflammation and can be useful not only in diagnosing autoimmune diseases such as rheumatoid arthritis but also in monitoring the disease process. It can be an index observing the activity and severity of RA synovitis. The combination of ESR and C- reactive protein (CRP) may improve sensitivity and specificity of the diagnosis of RA [9]. This examination expected to estimation IL-33 with it is receptor IL$1 \mathrm{R} 4$ levels, and assurance IL-33/IL-1R4 proportion as biomarker to atherosclerosis risk in rheumatoid arthritis Iraqi females' patients with and without dyslipidemia.

\section{Experimental}

\subsection{Patients' groups Study}

This study was attemptedat Baghdad Teaching Hospital, and included 60 female's patients with rheumatoid arthritis that were partitioned into: 30 patients with dyslipidemia(G2) and 30 patients without dyslipidemia(G3), in addition to 30 individuals as control gathering (G1). Patients were experiencing treatment by methortexiene medication, and they were analyzed by clinical examination by specialists, RF and ESR tests. All patients and control bunches age extended from (30-55) years. 


\subsection{Determination of serum RF}

The RF-Latex Test is a fast agglutination system, in view of change of the Singer method1 created for the immediate recognition, the semi-quantitation on a slide of rheumatoid factor (RF) in serum. The test is performed by testing a suspension of latex particles covered with human gamma globulin against obscure serums. The nearness or nonattendance of an unmistakable agglutination, demonstrates the presence or nonappearance of RF in the examples tried [10].

\subsection{Determination of ESR $(\mathrm{mm} / \mathrm{hr})$}

The Westergren technique is suggested as the reason for a satisfactory standard for ESR test, which that measures the sedimentation rate of totaled red cells in plasma. The numerical incentive in $\mathrm{mm}$ is acquired by estimating the separation between the least purpose of the surface meniscus to the furthest reaches of the red cell dregs in a segment of anticoagulated and weakened blood that has remained in a chosen tube for a time of an hour [11].

\subsection{Determination of serum Interleukin 1 Receptor 4(IL-1R4) (ng/ml)}

The IL-1R4 was controlled by utilizing the focused compound - connected invulnerable sorbent test (ELISA) pack that was provided by My Bio Source, USA, which that in light of strategy using a polyclonal hostile to IL-1R4 counter acting agent and an IL-1R4-HRP conjugate.

\subsection{Determination of serum IL-33 (pg/ml)}

Serum IL-33 levels were estimated utilizing particular protein connected immunosorbent test (ELISA) unit (Ray Bio Human IL-33 for in vitro quantitative estimation of human IL33 in serum), as indicated by the fabricates convention [12].

\subsection{Statistical Analysis}

Data were communicated as (Mean \pm SEM). The examination amongst patients and control group was communicated by t-test. T-test of $<0.005,<0.05$, and $<0.05$ were considered exceptionally highly significant, significant, and non-significant respectively. Pearson's relationship coefficient $(r)$ is utilized for depicting the relationship between the diverse examination parameters.

\section{Results and Discussion}

The levels of RF, ESR, IL-1R4, IL-33, and IL-33/IL-1R4 ratio levels in control (G1), rheumatoid arthritis female's patients with dyslipidemia (G2) and without dyslipidemia (G3) groups were summarizes in Table 1. The results which expressed as (mean $\pm \mathrm{SEM}$ ), showed a highly significant increase $(\mathrm{p}<0.005)$ in rheumatoid factor RF and ESR levels in both G2 and (G3) patients groups, respectively, when comparing with control group (G1), while there were no significant different ( $p>0.05$ ) in RF and ESR levels between two patients' groups. RF is autoantibodies coordinated against the fragment crystallizable (Fc) region of $\operatorname{IgG}$. Rheumatoid factor is an entrenched analytic and prognostic test in Rheumatoid Arthritis. High titer IgM RF is moderately particular for the finding of RA with regards to an interminable polyarthritis, and was for quite a long time the sole serologic measure broadly utilized as a part of the determination of RA. Patients with RA take after a variable infection course as to 
outcome measures, for example, utilitarian status or radiological appraisal of joint damage [13]. Aggravation in the synovium is reflected by a foundational provocative reaction. Expanded ESR and hepatocyte creation of intense stage proteins including CRP and intense stage serum amyloid an (A-SAA) are surrogate markers of this procedure [14]. In clinical management of RA, CRP and ESR are commonly ordered tests to guide diagnosis of RA besides the measurements of the mentioned auto antibodies. ESR is a nonspecific measure of inflammation and can be useful not only in diagnosing autoimmune diseases, such as rheumatoid arthritis, but also in monitoring the disease process. It can be an index observing the activity and severity of RA synovitis [15].

The results in the current study show a highly significant $(\mathrm{p}<0.005)$ elevation in IL-1R4 and IL-33 levels in both of G2 and G3 patients when compared with control group (G1), and there was no significant different $(\mathrm{p}>0.05)$ in IL-1R4 level and a significant different $(\mathrm{p}<$ 0.05 ) in IL-33 level between G2 and G3 patients.

Also, the results in Table 1 revealed to no significant decrease ( $>$ > 0.05) in IL-33/IL-1R4 ratio levels in both of G2 and G3 patients when compared with control group (G1). The present study showed a highly significant decrease $(\mathrm{p}<0.005)$ in AIP levels in both of G2 and G3 patients when compared with control (G1).

IL-33 can be discharged extracellularly as full-length protein through cell passing pathways and it can be cut to shorter structures with expanded cytokine movement. Going about as a cytokine and authoritative to its receptor IL-1R4 (previously known as T1/ST2), IL-33 drives compose 2-subordinate irritation and furthermore tissue repair, and it is associated with unfavorably susceptible and lung/mucosal aggravation. The dissolvable type of the receptor, sST2/sIL-1R4, is a characteristic inhibitor of IL-33 [16, 17]. In necrosis, the IL-33/ST2 pathway has been demonstrated to be a critical signaling pathway inducing related immune mechanisms, while IL-33/sST2 appeared to be an interesting pathway in neutralizing the activation of IL-33 [18].

Numerous reports have discovered expanded IL-33 and ST2 generation in the serum and synovial tissue of patients with RA. The articulation level of IL-33 and ST2 are seems to be correspond with RA sickness movement.Organization of IL-33 prompts the generation of expert provocative cytokines (IL-1 $\beta$, MCP-1 and IL-6) and fuels the advancement of CIA in mice. This information show that revocation of IL-33/ST2 opened up incendiary reaction speaks to a restorative focus for RA [19]. The declaration of sST2 is corresponded with the continuous procedure of fibrosis and inflammation. After intense myocardial localized necrosis, the IL-33 level is not quite the same as the sST2 level, which shows that the control of IL-33 and sST2 is diverse after intense myocardial dead tissue. Mineralocorticoid receptor enemies have been appeared to lessen cardiovascular fibrosis through balancing IL-33/ST2 and galectin-3 flagging.These outcomes show that IL-33/ST2 assumes a defensive part in cardiovascular fibrosis, and sST2 adversely directs this pathway as a bait receptor for IL-33 [20].

It has been demonstrated that the receptor of IL-33 assumes to be a critical part in aggravation, contamination, and immune system infections. Recently, IL-33has been appeared to change the manifestations of rheumatoid joint pain, foundational lupus erythematosus, and other immune system ailments. They may inspire gainful or hindering impacts relying upon the ailment setting. Along these lines, ponders on IL-33may give another thought and focus for the treatment of immune system illnesses [21]. As a kind of 
pro-inflammatory cytokines, IL-33 cause the immune pathological damage in some tissues. It plays the important role in chronic inflammatory and autoimmune diseases. It has been demonstrated that IL-33/ST2 flag transduction pathways are associated with different obsessive procedures.IL-33 and its receptor ST2 are the potential focuses for the treatment of hypersensitive and immune system fiery maladies [22].

Large amounts of sST2 can predict worse prognosis in cardiovascular patients, which proposes that sST2 levels are adequate to sequester endogenous IL-33 levels. In spite of the present absence of data on endogenous IL-33 levels in people [23].

The essential site of irritation in RA is the synovial tissue, from which cytokines are discharged into the fundamental course. These flowing cytokines are in a situation to adjust the working of fat tissue, skeletal muscle, liver, and vascular endothelium and produce a range of proatherogenic changes that incorporates insulin opposition, a trademark dyslipidemia, prothrombotic impacts, ace oxidative pressure and endothelial brokenness [24].

RA patients will probably create atherosclerotic plaques, quiet ischemic sickness and they have an expanded danger of sudden heart passing, contrasted with the general population [25]. In patients with RA, the danger of CVD is expanded and screening of CVD risk factors and distinguishing proof of high-chance patients is justified [26].

Study show that CRP and ESR have been related with CVD in RA and polyarthritis [27]. Determinants of provocative action, other 'ailment related' markers have been recognized as potential novel hazard factors for CVD in RA, notably RF energy and anti-citrullinated protein antibody (ACPA) positivity [28].

In this study, Figures 1. and 3. were showed a highly significant positive correlation between IL-1R4 with both of RF in G1( $r=0.155), G 2(r=0.054)$, and G3( $r=0.008)$, and Figure 4 show a highly significant positive correlation with IL-33 in G1 ( $\mathrm{r}=0.145), \mathrm{G} 2(\mathrm{r}=$ $0.078)$, and $\mathrm{G} 3(\mathrm{r}=0.148)$, respectively. Also in Figure 2., there were a highly significant negative correlation between IL-1R4 with ESR in G1(r=-0.147) and G3 ( $\mathrm{r}=-0.441)$, and a highly significant positive correlation with ESR in G2(r =0.108). The results in Figure 3. revealed no significant negative correlation between IL-1R4 and IL-33/IL-1R4 ratio in both of $\mathrm{G} 1(\mathrm{r}=-0.345), \mathrm{G} 2(\mathrm{r}=-0.338)$ and $\mathrm{G} 3(\mathrm{r}=-0.688)$. 
Table 1. Levels of rheumatoid factor (RF), Erythrocyte sedimentation. rate (ESR), Interleukin 1 Receptor 4(IL1R4), IL-33, and IL-33/IL-1R4 proportion in charge (G1), rheumatoid arthritisfemales patients with dyslipidemia (G2) and without dyslipidemia (G3) groups.

\begin{tabular}{|l|l|l|l|l|l|l|}
\hline \multicolumn{1}{|c|}{ Groups } & \multicolumn{1}{|c|}{$\begin{array}{c}\text { G1 } \\
\text { No.(30) }\end{array}$} & \multicolumn{1}{|c|}{$\begin{array}{c}\text { G2 } \\
\text { No.(30) }\end{array}$} & $\begin{array}{c}\text { G3 } \\
\text { No.(30) }\end{array}$ & $\begin{array}{c}\text { P-value } \\
\text { G1\&G2 }\end{array}$ & $\begin{array}{c}\text { P-value } \\
\text { G1\&G3 }\end{array}$ & $\begin{array}{c}\text { P-value } \\
\text { G2\&G3 }\end{array}$ \\
\hline RF ( IU/mL) & $9.81 \pm 1.79$ & $83.73 \pm 15.3$ & $88.53 \pm 16.18$ & $H S$ & $H S$ & NS \\
\hline E.S.R (mm/hr) & $5.2 \pm 0.95$ & $41.83 \pm 6.64$ & $18.25 \pm 5.86$ & $H S$ & $H S$ & NS \\
\hline IL-1R4(ng/ml) & $0.29 \pm 0.05$ & $1.46 \pm 0.26$ & $1.62 \pm 0.29$ & $H S$ & $H S$ & NS \\
\hline IL33(pg/ml) & $136.46 \pm 24.9$ & $541.03 \pm 98.99$ & $483.7 \pm 88.42$ & $H S$ & $H S$ & S \\
\hline IL33 / IL1R4 & $479.44 \pm 87.6$ & $339.82 \pm 62.12$ & $344.93 \pm 63.5$ & NS & NS & NS \\
\hline
\end{tabular}

HS: $P<0.005$; S: $P<0.05$; NS: $P>0.05$

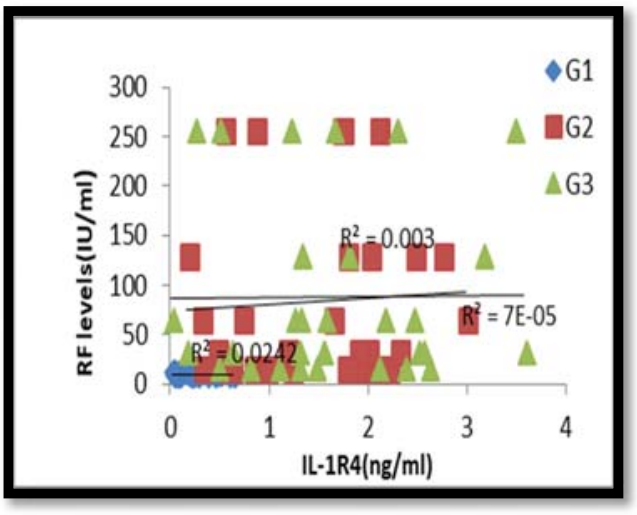

Figure 1. correlation between IL-1R4 and RF in G1, G2 and G3.

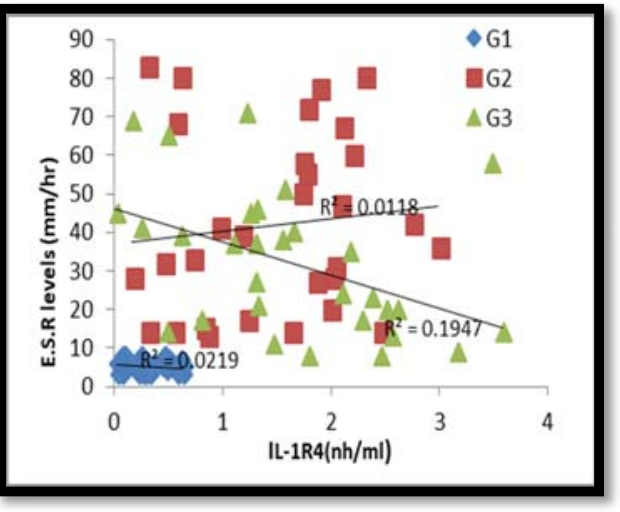

Figure 2. correlation between IL-1R4 and ESR in G1,G2 and G3. 


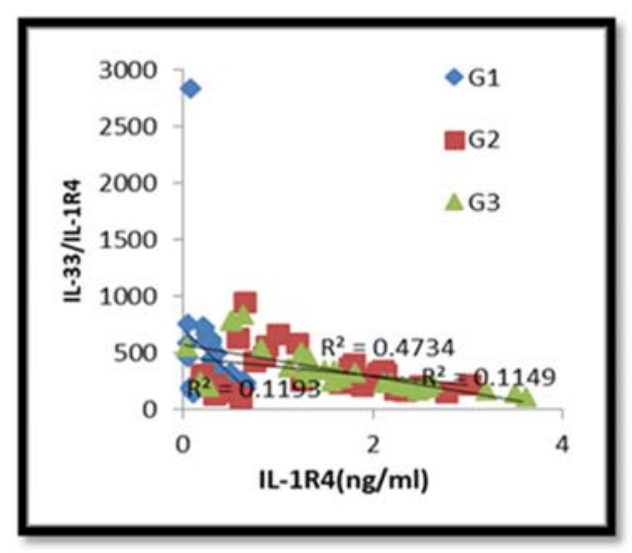

Figure 3. correlation of IL-1R4 and IL-33/IL-1R4 ratio in $\mathrm{G} 1, \mathrm{G} 2$, and $\mathrm{G} 3$.

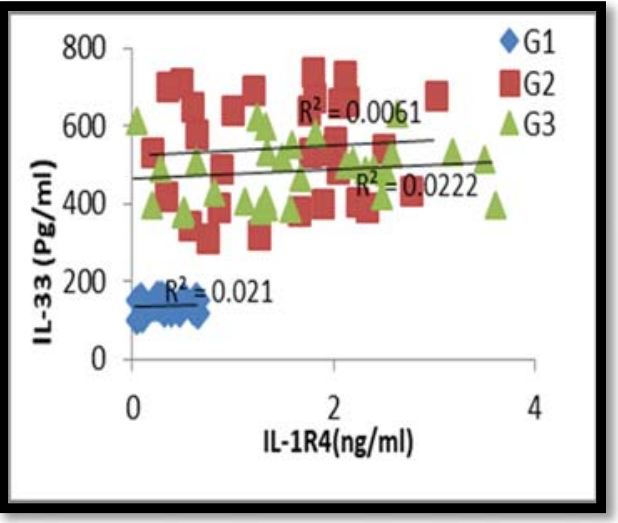

Figure 4. correlation of IL-1R4 and IL-33 in $\mathrm{G} 1, \mathrm{G} 2$, and $\mathrm{G} 3$.

\section{Conclusions}

In conclusion, current study proved a highly significant elevation in IL-1R4 and IL-33in addition to no significant decrease in IL-33/IL-1R4 ratio levels in Iraqi women patients with rheumatoid arthritis. In addition to the level of ESR, and IL-33 in rheumatoid arthritis Iraqi women patients with dyslipidemia were higher than that in rheumatoid arthritis Iraqi women patients without dyslipidemia, while the level of IL-33/IL-1R4 ratio in RA Iraqi women patients with dyslipidemia was lower than that in RA Iraqi women patients without dyslipidemia patients; therefore the RA women patients with dyslipidemia may be they are more prone to atherosclerosis and heart diseases from RA women patients without dyslipidemia.Although IL-1R4 (ST2) is a potentially useful biomarker in heart diseases such as heart failure and myocardial infarction. Recently, physicians have begun to use SST2 as a biomarker of cardiomyocyte stress and fibrosis, and the level of sST2 is now used for additional risk stratification of acute or ambulatory heart-failure patients.

Here we showed that the recently described ligand IL-33 was synthesized by cardiac fibroblasts and abrogated angiotensin II- and phenylephrine-induced hypertrophy in cardiomyocytes in vitro. Finally, it is possible that sST2 is not only a biomarker for poor outcome, but also a true pathophysiological mediator of disease progression. IL-33 has now also been shown to participate in cardiovascular pathophysiology. Furthermore, the IL-33/ST2 system may play a part in the progression of atherosclerotic vascular disease.

\section{References}

1. Malda, J.; Boere, J.; Van De Lest, C. H.; Van Weeren, P. R.; Wauben, M. H. Extracellular vesicles - new tool for joint repair and regeneration. Nature Reviews Rheumatology 2016, 12, 243-249.

2. Picerno, V.; Ferro, F.; Adinolfi , A.;Valentini, E.; Tani, C.; Alunno, A. One year in review: the pathogenesis of rheumatoid arthritis. Clinical and experimental rheumatology. 2015, 33, 551-558.

3. Alghasham, A.; Rasheed, Z. Therapeutic targets for rheumatoid arthritis: Progress and promises. Autoimmunity. 2014,47, 77-94. 
4. Townsend, M. J.; Fallon, P. G.; Matthews, D. J.; Jolin, H. E.; McKenzie, A. N. T1/ST2-deficient mice demonstrate the importance of T1/ST2 in developing primary T helper cell type 2 responses. Journal of experimental medicine 2000, 191,6, 1069-1076.

5. Jo, S.; Kim, E.; Kwak, A.; Lee, J.; Hong, J.; Lee, J.; Youn, S.; Bae, S.; Kim, B.; Ryoo, S.; Kang, T.B. Reconstitution of ST2 (IL-1R4) specific for IL-33 activity; no suppression by IL-1Ra though a common chain IL-1R3 (IL-1RAcP) shared with IL-1. Cytokine 2016, 83, 33-40.

6. Weinberg, E.O.; Shimpo, M.; De Keulenaer, G.W.; MacGillivray, C.; Tominaga, S.I.; Solomon, S.D.; Rouleau, J.L.; Lee, R.T. Expression and regulation of ST2, an interleukin-1 receptor familymember,in cardiomyocytes and myocardial infarction. Circulation. 2002, 106, 2961-2966.

7. Pascual-Figal. D.A.; Januzzi. J.L. The biology of ST2: The international ST2 consensus panel. The American journal of cardiology. 2015, 115, 3B-7B.

8. Schmitz, J.; Owyang, A.; Oldham, E.; Song, Y.; Murphy, E.; McClanahan, T.K.; Zurawski, G.; Moshrefi, M.; Qin, J.; Li, X.; Gorman, D.M. IL-33, an interleukin-1-like cytokyne that signals via the IL-1 receptor-related protein ST2 and induces T helper type 2-associated cytokynes. Immunity. 2005, 23, 479-490.

9. Kakkar, R.; Lee, R.T. The IL-33/ST2 pathway: therapeutic target and novel biomarker. Nature reviews Drug discovery. 2008, 7, 827-840.

10. Singer, J.M.; Plotz, C.M. The latex fixation test. I. Application to the serologic diagnosis of rheumatoid arthritis. American Journal of Medicine. 1956, 21, 888-892.

11. Boroviczeny, K-G.V.; Bottiger, L. E.; Chattis, A. J. B.; Dawson. K.; Fukutake. F. W.; Gunz, S. M. Lewis.; Rewald, E. M.; Roubicek, G.; Ruhenstroth-Bauer.; and Westergren, A. Reference method for the erythrocyte sedimentation rate (ESR) test on human blood. Journal of Clinical Pathology. 1973, 26, 301-302

12. Burtis, CA.; Ashwood, ER.; Bruns, DE.; Tietz. Textbook of Clinical Chemistry and Molecular Diagnostics, ${ }^{\text {th }}$ ed, Elsevier Saunders, 2006.

13. Spartali, I.; Kostantinos, H.; Ioannis, K.;Thrasivoulos, P. Body fat percentage and body mass index as predictors of cadets' physical performance. The Open Sports Sciences Journal. 2014, 7,53-59.

14. Ingegnoli, F.; Castelli, R.;Gualtierotti, R. Rheumatoid factors: clinical applications. Disease marker.s 2013, 35, 727-734.

15. Nielen, M.M.; Van Schaardenburg, D.; Reesink, H.W.; Twisk, J.W.; Van De Stadt, R.J.; Van Der Horst-Bruinsma, I.E.; De Gast, T.; Habibuw, M.R.; Vandenbroucke, J.P.; Dijkmans, B.A. Increased levels of C-reactive protein in serum from blood donors before the onset of rheumatoid arthritis. Arthritis \& Rheumatism: Official Journal of the American College of Rheumatology. 2004, 50, 2423-2427.

16. Shadick, N.A.; Cook, N.R.; Karlson, E.W.; Ridker, P.M.; Maher, N.E.; Manson, J.E.; Buring, J.E.; Lee, I.M. C-reactive protein in the prediction of rheumatoid arthritis in women. Archives of Internal Medicine. 2006, 166, 2490-2494.

17. Gordon, E.D.; Simpson, L.J.; Rios, C.L.; Ringel, L.; Lachowicz-Scroggins, M.E.; Peters, M.C.; Wesolowska-Andersen, A.; Gonzalez, J.R.; MacLeod, H.J.; Christian, L.S.; Yuan, S. Alternative splicing of interleukin-33 and type 2 inflammation in asthma. Proceedings of the National Academy of Sciences. 2016, 113, 8765-8770. 
18. Finlay, C. M.; Stefanska, A. M.; Walsh, K. P. Helminth products protect against autoimmunity via innate type 2 cytokines IL-5 and IL-33, which promotes eosinophilia, The Journal of Immunology. 2016, 196, 703-714.

19. Sanada, S.; Hakuno, D.; Higgins, L. J.; Schreiter, E. R.; McKenzie, A. N.; Lee, R. T. IL-33 and ST2 comprise a critical biomechanically induced and cardioprotective signaling system. The Journal of clinical investigation. 2007, 117, 1538-1549.

20. Hong, Y.S.; Moon, S.J.; Joo, Y.B.; Jeon, C.H.; Cho, M.L.; Ju, J.H.; Oh, H.J.; Heo, Y.J.; Park, S.H.; Kim, H.Y.; Min, J.K. Measurement of interleukin-33 (IL-33) and IL-33 receptors (sST2 and ST2L) in patients with rheumatoid arthritis. Journal of Korean medical science. 2011, 26, 1132-1139.

21. Lax, A.; Sanchez-Mas, J.; Asensio-Lopez, M.C.; Fernandez-Del Palacio, M.J.; Caballero, L.; Garrido, I.P.; Pastor-Perez, F.J.; Januzzi, J.L.; Pascual-Figal, D.A. Mineralocorticoid receptor antagonists modulate galectin-3 and interleukin-33/ST2 signaling in left ventricular systolic dysfunction after acute myocardial infarction. JACC Heart Fail. 2015, 3, 50-58.

22. Seltmann, J.; Werfel, T.; Wittmann, M. Evidence for a regulatory loop between IFN- $\gamma$ and IL-33 in skin inflammation. Experimental dermatology. 2013, 22, 102-107.

23. Zhao, Q.; Chen, G. Role of IL-33 and Its Receptor in T Cell-Mediated Autoimmune Diseases. BioMed Research International. 2014, 2014, 1-10, doi.org/10.1155/2014/587376.

24. Shimpo, M.; Morrow, D.A.; Weinberg, E.O.; Sabatine, M.S.; Murphy, S.A.; Antman, E.M.; Lee, R.T. Serum levels of the interleukin-1 receptor family member ST2 predict mortality and clinical outcome in acute myocardial infarction. Circulation. 2004, 109, 2186-2190.

25. McInnes, I.B. Rheumatoid arthritis: from bench to bedside. Rheumatic Disease Clinics of North America. 2001, 27, 373-387.

26. Ogdie, A.; Yu, Y.; Haynes, K.; Love, T.J.; Maliha, S.; Jiang, Y.; Troxel, A.B.; Hennessy, S.; Kimmel, S.E.; Margolis, D.J.; Choi, H. Risk of major cardiovascular events in patients with psoriatic arthritis, psoriasis and rheumatoid arthritis: a population-based cohort study. Annals of the rheumatic diseases. 2015, 74, 326-332.

27. Avina-Zubieta, J.A.; Thomas, J.; Sadatsafavi, M.; Lehman, A.J.; Lacaille, D. Risk of incident cardiovascular events in patients with rheumatoid arthritis: a meta-analysis of observational studies. Annals of the rheumatic diseases. 2012, 71, 1524-1529.

28. Aubry, M.C.; Maradit-Kremers, H.; Reinalda, M.S.; Crowson, C.S.; Edwards, W.D.; Gabriel, S.E. Differences in atherosclerotic coronary heart disease between subjects with and without rheumatoid arthritis. The Journal of rheumatology. 2007, 34, 937-942. 